\title{
Synthesis and Fungicidal Activities of Novel 1,2,3,4-Substituted-Furans Derivatives
}

\author{
Yanlin Zhang', Zhihui Zou', Hua Cao ${ }^{2, *}$ \\ ${ }^{1}$ Department of Environmental Monitoring, Guangdong Vocational College of Environmental Protection Engineering, Foshan, China \\ ${ }^{2}$ School of Chemistry and Chemical Engineering, Guangdong Pharmaceutical University, Guangzhou, China
}

Email address:

zylin1980@126.com (Yanlin Zhang), gwsyzx@gdpu.edu.cn (Hua Cao)

${ }^{*}$ Corresponding author

To cite this article:

Yanlin Zhang, Zhihui Zou, Hua Cao. Synthesis and Fungicidal Activities of Novel 1,2,3,4-Substituted-Furans Derivatives. American Journal of Heterocyclic Chemistry. Vol. 4, No. 3, 2018, pp. 42-48. doi: 10.11648/j.ajhc.20180403.11

Received: November 18, 2018; Accepted: December 4, 2018; Published: December 17, 2018

\begin{abstract}
It is well known that furans derivatives have high biological activities and commercialized furans compounds have extensive applications in life science and medicine field, however, the studies on its potential fungicidal activities are rare and lack of reports. In order to find novel candidate compounds with high fungicidal efficiency, a series of furans derivatives were synthesized and their fungicidal activities were evaluated, which provides information for molecular design and modification of furans compounds with highly effective broad-spectrum fungicidal activities. A series of novel 1,2,3,4-substituted-furans derivatives were synthesized by one-pot method and the structures were confirmed by ${ }^{1} \mathrm{H}$ NMR and ${ }^{13} \mathrm{C}$ NMR. The fungicidal activities were evaluated by the mycelium growth rate method in vitro. Compound c7, c14, c15, c17 and c18 against Fusarium oxysporum had comparable activities with chlorothalonil. Among them, compound $\mathrm{c} 14$ and c15 against $F$. oxysporum with $\mathrm{EC}_{50}$ value of $14.71 \mathrm{mg} / \mathrm{L}$ and $14.39 \mathrm{mg} / \mathrm{L}$, respectively, which were superior to that of chlorothalonil. Dimethyl 5-methyl-4-(2-phenylethynyl) furan-2,3-dicarboxylate deserved further development as one kind of novel promising fungicidal agents.
\end{abstract}

Keywords: Furans Derivatives, Fungicidal Activities, Nano- $\mathrm{Cu}_{2} \mathrm{O}-$ Catalyzed Synthesis

\section{Introduction}

Furans derivatives are a fundamental class of oxo five-membered hererocyclic compounds, furan rings exist in many natural products and drug molecules widely [1-3]. Because its privileged skeleton could be optimized by bearing many active substituted groups, substituted furans play an important role in organic molecular design. More importantly, substituted-furan derivatives exhibit extensive bioactivities and pharmacological activities, which have become important drug molecules structural units and synthetic intermediates [4-6].

As shown in Figure 1, furan derivatives were put on the market and now more than 10 furan derivatives commercially available. Among the marketed drugs, ranitidine (i) was effectively applied in the treatment of peptic ulcer by inhibiting gastric acid secretion [7, 8] and furosemide (ii) was used widely as loop diuretics to treat congestive heart failure and edema $[9,10]$.

Moreover, another pharmacological activities of furan drugs were antibacterial, and the most commonly-used broad-spectrum antibacterial anti-inflammatory drug was nitrofuran antibiotics including furazolidone (iii) [11, 12], nitrofurazone (iv) [13], nitrofurantoin (V) [14] and furaltadone (vi) [15], which have been widely used in aquaculture and livestock raising for the prevention and treatment of enteritis, scabies, red fin disease and ulcers caused by Escherichia coli or Salmonella infection.

About pesticidal activities, some furan derivatives exhibit efficient insecticidal activity, for instance, carbofuran (VII), once was widely used as a nematicide on soybeans, rice, potatoes, fruits and vegetables, however, the use of carbofuran has been restricted at present in many regions [16].

Furan derivatives, the subject of extensive attention in pesticide and medicine fields, worth the attention for many reasons: it is not only extensively used as synthetic building blocks for the synthesis of more complex compounds, but also 
as communicating moieties in molecular materials [17]. Recently, organic synthesis chemists have been making extensive efforts to construct furans derivatives by developing new and efficient synthetic transformations [18-21].

Although several useful procedures have been developed which allow the preparation of functionalized furan molecules, there only few chemists and biologists interested in application those novel method on bioactivity. In the study, we not only designed and synthesized a series of functionalized furan compounds by optimization of synthetic routes and reaction conditions, but also explored their fungicidal activities, which is of great significance to further study on structure-activity relationships and screening for efficient broad-spectrum lead compounds with low toxicity.<smiles>CN/C(=C/[N+](=O)[O-])NCCSCc1ccc(CN(C)C)o1</smiles>

(Z)- $N$-(2-(((5-((dimethylamino)methyl)furan-2-yl)methyl)thio)ethyl)- $N$-methyl-2nitroethene-1,1-diamine

(i)<smiles>NS(=O)(=O)c1cc(C(=O)O)c(NCc2ccco2)cc1Cl</smiles>

4-chloro-2-((furan-2-ylmethyl)amino)-5-sulfamoylbenzoic acid

(ii)<smiles>O=C1OCCN1/N=C/c1ccc([N+](=O)[O-])o1</smiles>

(E)-3-(((5-nitrofuran-2-yl)methylene)amino)oxazolidin-2-one

(iii)<smiles>O=C1CN(/N=C/c2ccc([N+](=O)[O-])o2)C(=O)N1</smiles>

(E)-1-(((5-nitrofuran-2-yl)methylene)amino)imidazolidine2,4-dione

(v)<smiles>NC(=O)N/N=C/c1ccc([N+](=O)[O-])o1</smiles>

(E)-2-((5-nitrofuran-2yl)methylene)hydrazinecarboxamide

(iv)<smiles>CNC(=O)Oc1cccc2c1OC(C)(C)C2</smiles>

2,2-dimethyl-2,3-dihydrobenzofuran-7-yl methylcarbamate

(vii)

Figure 1. Substituted-furans derivative with different biological activities. 


\section{Materials and Methods}

\subsection{Materials}

\subsubsection{Plant Pathogen}

All the compounds were tested for their fungicidal activities against five phytopathogenic fungi including Fusarium oxysporum, Alternaria solani, Fusarium graminearum, Rhizoctonia solani and Valsa mali, which were supplied by Guangdong Institute of Microbiology. The tested pathogenic fungi were inoculated in potato dextrose agar medium (PDA) at $37^{\circ} \mathrm{C}$ for $3 \sim 7 d$.

\subsubsection{Instruments and Reagents}

Bruker Avance III Nuclear magnetic resonance spectrometer (TMS, $\mathrm{CDCl}_{3}$ ). DABCO, dichloromethane, alkyne, alkynyl alcohol, toluene, nano- $\mathrm{Cu}_{2} \mathrm{O}, \mathrm{AgOAc}$ and triphenylphosphin, ether, hexane, ethyl acetate were commercially available analytical reagent. Chlorothalonil was used as control fungicide and the purity is $97.12 \%$, which was made from Jiangsu Ruibang Pesticide Factory Co., Ltd.

\subsection{Synthesis and Characterization of Target Compounds}

\subsubsection{Synthetic Route}

The construction of furan derivatives with structurally diverse substituted groups are an effective way to obtain new heterocyclic derivatives with high fungicidal. The strategy of the synthetic approach is convenient and simple to construct different poly-substituted furan compounds (c1-c26) in the presence of nano- $\mathrm{Cu}_{2} \mathrm{O}$ or $\mathrm{AgOAc}$, respectively (Figure 2).

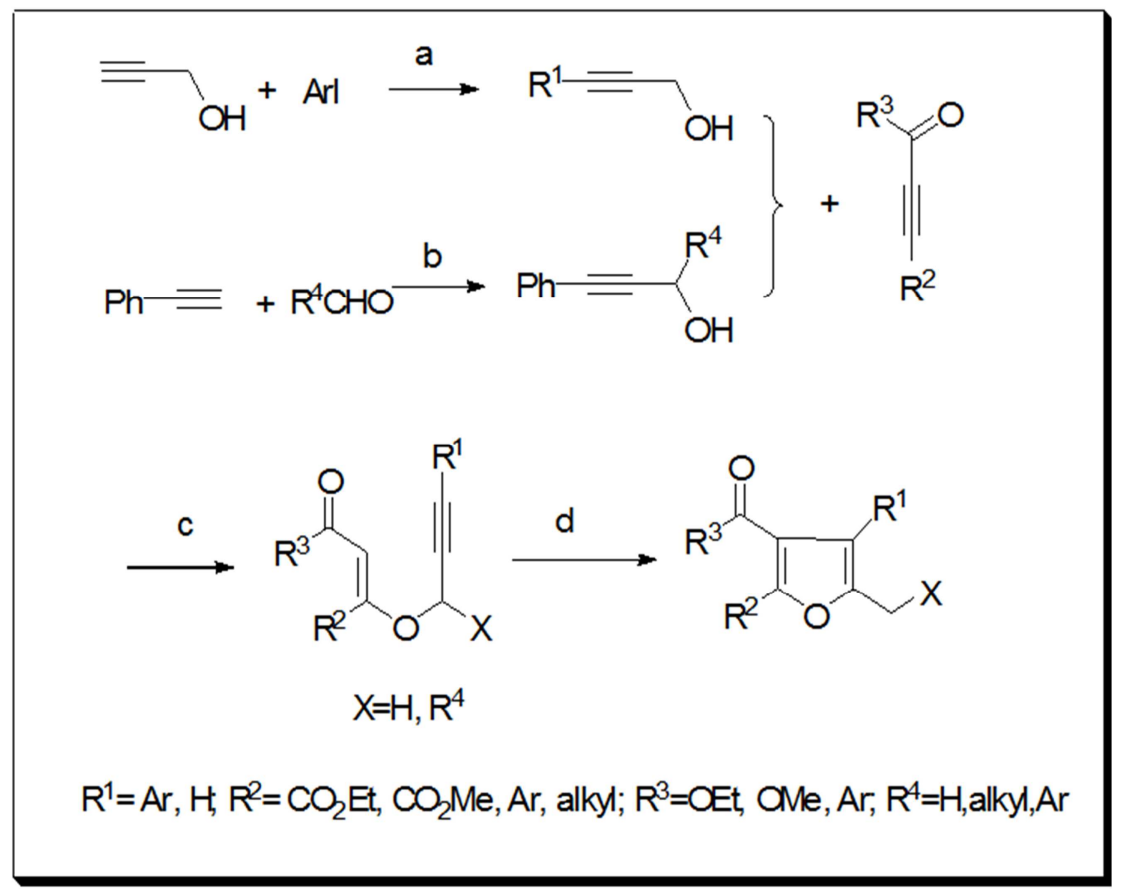

Figure 2. Regents and Conditions: a) $\mathrm{PdCl}_{2}, \mathrm{CuI}, \mathrm{NEt} t_{3}, \mathrm{THF}, \mathrm{rt}$; b) $\mathrm{BuLi},-78^{\circ} \mathrm{C}$; c) $\mathrm{DABCO} /, \mathrm{CH}_{2} \mathrm{Cl}_{2}, \mathrm{rt}$; d) $\mathrm{AgOAc}, \mathrm{PPh}_{3}, \mathrm{DMF}, 50^{\circ} \mathrm{C}$.

\subsubsection{Preparation of Furans Derivatives}

The novel 1,2,3,4-substituted-furans derivatives were synthesized with DABCO as catalyst via nucleophilic addition reaction in dichloromethane. The mixture of alkyne and alkynyl alcohol was added to dichloromethane in a round bottom flask under stiring and then a catalyst amount of DABCO was added. After reacting for $10 \mathrm{~min}$, the solvent was evaporated, and then $2-3 \mathrm{ml}$ of toluene, AgOAc and triphenylphosphine were added into the round bottom flask. The reaction mixture was stirred at $50^{\circ} \mathrm{C}$ until reaction was over. A yellow mixture obtained was evaporated under reduced pressure and extracted with ether-water solution $(1: 1$, $V / V)$, and the residue was washed with ultrapure water $(20$ $\mathrm{ml} \times 3$ ). The reaction mixture extracted by ether was dried by anhydrous sodium sulfate and concentrated by rotary vacuum evaporation. The crude product was finally separated and purified by column chromatography with hexane-ethyl acetate
$(6: 1, \mathrm{~V} / \mathrm{V})$

\subsection{Bioassay}

The preliminary fungicidal activities were evaluated by the mycelium growth rate method in vitro at the concentration of $50 \mathrm{mg} / \mathrm{L}$. All the compounds were tested for their fungicidal activities against five phytopathogenic fungi including Fusarium oxysporum, Alternaria solani, Fusarium graminearum, Rhizoctonia solani and Valsa mali. Chlorothalonil, a commercial fungicide, was used as the positive control. The synthesized compounds and positive control were dissolved thoroughly in dimethyl sulfoxide to prepare $20 \mathrm{~g} / \mathrm{L}$ stock solutions, which were diluted to tested solutions at concentration of $500 \mathrm{mg} / \mathrm{L}$ with $0.1 \%$ Tween- 80 solution before test. A given volume of tested agent was mixed with sterilized PDA medium at about $55^{\circ} \mathrm{C}$ in a ratio of $1: 9$ $(v / v)$ containing $50 \mathrm{mg} / \mathrm{L}$ tested compounds. Then the fresh 
fungus cakes with diameter of $4 \mathrm{~mm}$ were inoculated onto the plate center with mycelium downward and were cultured at $27^{\circ} \mathrm{C}$. Until mycelium growing up to two-thirds of plates, the diameters of fungus mycelia were measured by cross method. The test had set up blank control group added no tested compound and was repeated in triplicate. The average diameters of fungus mycelia were used to calculate inhibition rate according to the formula: $I=(D \mathrm{c}-D \mathrm{t}) /(D \mathrm{c}-0.4) \times 100 \% . I$ was inhibition rate, $D c$ and $D t$ were the average diameters of mycelia in the blank control group and compound treatment groups, respectively.

In addition, $\mathrm{EC}_{50}$ value of compounds with potential fungicidal activities against $F$. oxysporum was further tested. Briefly, the plates containing compound at various concentrations were prepared by the two-fold agar dilution method and final concentration of the test compound in the culture medium was 100.0, 50.0, 25.0, 12.5, 6.25 and 3.125 $\mathrm{mg} / \mathrm{L}$, respectively. The relationship between the probability value of inhibition rate and logarithm of compound concentrations was explored by regression analysis, and obtained toxicity regression equations, $\mathrm{EC}_{50}$ value and its with $95 \%$ confidence interval.

\section{Results and Discussion}

\subsection{Chemistry}

According to the synthetic route, a series of new type furans derivatives were designed, synthesized and characterized with ${ }^{1} \mathrm{H}$ NMR and ${ }^{13} \mathrm{C}$ NMR spectra (Table 1). As shown in Table 2, various electron-deficient alkynes with 2 -yn-1-ols underwent this process smoothly in moderate to good yields (20\%-79\%) in the presence of air under atmospheric pressure. These results indicated that this transformation tolerates functional groups including the electron-rich and electron-withdrawing groups at different positions of the aromatic ring. Notably, no other regioisomers were detected, which was indicated that this cyclization was regioselective and chemoselective.

Table 1. ${ }^{1} \mathrm{H} N \mathrm{NR}\left(400 \mathrm{MHz}, \mathrm{CDCl}_{3}\right)$ and ${ }^{13} \mathrm{C} \mathrm{NMR}(100 \mathrm{MHz}, \mathrm{CDCl}$ ) data of target compounds.

\begin{tabular}{|c|c|c|}
\hline Compounds & ${ }^{1} \mathrm{H} N M R, \boldsymbol{\delta}_{\mathrm{H}}$ & ${ }^{13} \mathrm{C}$ NMR, $\delta$ \\
\hline $\mathrm{c} 1$ & $6.30(\mathrm{~s}, 1 \mathrm{H}), 4.25-4.31(\mathrm{~m}, 4 \mathrm{H}), 2.30(\mathrm{~s}, 3 \mathrm{H}), 1.26-1.32(\mathrm{~m}, 6 \mathrm{H})$ & $\begin{array}{l}162.7,157.8,155.4,142.0,125.1,109.2,61.2,14.1,14.0 \\
13.5\end{array}$ \\
\hline c2 & $4.27-4.35(\mathrm{~m}, 4 \mathrm{H}), 2.25(\mathrm{~s}, 3 \mathrm{H}), 1.96(\mathrm{~s}, 3 \mathrm{H}), 1.29-1.35(\mathrm{~m}, 6 \mathrm{H})$ & $\begin{array}{l}163.9,157.9,152.0,140.0,126.5,116.57,61.3,61.0,14.2, \\
14.1,11.7,8.50\end{array}$ \\
\hline c3 & $\begin{array}{l}4.28-4.36(\mathrm{~m}, 4 \mathrm{H}), 2.39(\mathrm{~d}, 2 \mathrm{H}, J=7.6 \mathrm{~Hz}), 2.27(\mathrm{~s}, 3 \mathrm{H}), 1.29-1.36(\mathrm{~m}, \\
6 \mathrm{H}), 1.07(\mathrm{t}, 3 \mathrm{H}, J=7.6 \mathrm{~Hz})\end{array}$ & $\begin{array}{l}164.1,157.9,151.8,139.7,122.8,110.8,61.4,61.0,16.9 \\
14.7,14.1,14.0,11.7\end{array}$ \\
\hline $\mathrm{c} 4$ & $\begin{array}{l}7.22-7.34(\mathrm{~m}, 5 \mathrm{H}), 4.29(\mathrm{q}, 2 \mathrm{H}, J=7.2 \mathrm{~Hz}), 4.21(\mathrm{q}, 2 \mathrm{H}, J=7.2 \mathrm{~Hz}), 2.34 \\
(\mathrm{~s}, 3 \mathrm{H}), 1.31(\mathrm{t}, 3 \mathrm{H}, J=7.2 \mathrm{~Hz}), 1.14(\mathrm{t}, 3 \mathrm{H}, J=7.2 \mathrm{~Hz})\end{array}$ & $\begin{array}{l}163.9,157.8,152.6,139.5,131.7,130.7,128.8,128.3,127.7, \\
126.6,122.6,111.4,61.6,61.2,14.2,13.9,12.6\end{array}$ \\
\hline c5 & $\begin{array}{l}7.19-7.21(\mathrm{~m}, 1 \mathrm{H}), 7.02-7.09(\mathrm{~m}, 3 \mathrm{H}), 4.31(\mathrm{q}, 2 \mathrm{H}, J=7.2 \mathrm{~Hz}), 4.21(\mathrm{q}, 2 \mathrm{H}, \\
J=7.2 \mathrm{~Hz}), 2.34(\mathrm{~s}, 3 \mathrm{H}), 2.30(\mathrm{~s}, 3 \mathrm{H}), 1.29(\mathrm{t}, 3 \mathrm{H}, J=6.8 \mathrm{~Hz}), 1.16(\mathrm{t}, 3 \mathrm{H}, \\
J=6.8 \mathrm{~Hz})\end{array}$ & $\begin{array}{l}164.0,157.8,152.6,139.4,138.1,130.6,129.4,128.5,128.4, \\
126.7,125.8,122.7,61.6,61.2,21.3,14.1,13.9,12.6\end{array}$ \\
\hline c6 & $\begin{array}{l}7.11-7.26(\mathrm{~m}, 4 \mathrm{H}), 4.39(\mathrm{q}, J=8.0 \mathrm{~Hz}, 2 \mathrm{H}), 4.14(\mathrm{q}, 2 \mathrm{H}, J=8.0 \mathrm{~Hz}), 2.21 \\
(\mathrm{~s}, 3 \mathrm{H}), 2.15(\mathrm{~s}, 3 \mathrm{H}), 1.37(\mathrm{t}, 3 \mathrm{H}, J=8.0 \mathrm{~Hz}), 1.08(\mathrm{t}, 3 \mathrm{H}, J=8.0 \mathrm{~Hz})\end{array}$ & $\begin{array}{l}163.3,157.9,152.7,140.0,137.4,130.4,129.9,128.3,126.9, \\
125.5,122.4,111.3,61.2,60.6,19.7,14.2,13.7,12.3\end{array}$ \\
\hline c7 & $\begin{array}{l}8.05(\mathrm{~d}, 2 \mathrm{H}, J=8.0 \mathrm{~Hz}), 7.34(\mathrm{t}, 2 \mathrm{H}, J=8.0 \mathrm{~Hz}), 4.37(\mathrm{q}, 2 \mathrm{H}, J=8.0 \mathrm{~Hz}), \\
4.26(\mathrm{q}, 2 \mathrm{H}, J=8.0 \mathrm{~Hz}), 3.90(\mathrm{~s}, 3 \mathrm{H}), 2.39(\mathrm{~s}, 3 \mathrm{H}), 1.33(\mathrm{t}, 3 \mathrm{H}, J=8.0 \mathrm{~Hz}), \\
1.19(\mathrm{t}, 3 \mathrm{H}, J=8.0 \mathrm{~Hz})\end{array}$ & $\begin{array}{l}\text { 166.6, 163.6, 157.6, 153.0, 152.9, 140.0, 135.5, 129.8, 128.8, } \\
126.1,121.8,61.8,61.4,52.1,14.1,13.9,12.7\end{array}$ \\
\hline $\mathrm{c} 8$ & $\begin{array}{l}7.22(\mathrm{~d}, 2 \mathrm{H}, J=8.0 \mathrm{~Hz}), 6.94(\mathrm{t}, 2 \mathrm{H}, J=9.6 \mathrm{~Hz}), 4.39(\mathrm{q}, 2 \mathrm{H}, J=8.0 \mathrm{~Hz}) \\
4.27(\mathrm{q}, 2 \mathrm{H}, J=8.0 \mathrm{~Hz}), 3.82(\mathrm{~s}, 3 \mathrm{H}), 2.39(\mathrm{~s}, 3 \mathrm{H}), 1.36(\mathrm{t}, 3 \mathrm{H}, J=7.2 \mathrm{~Hz}) \\
1.24(\mathrm{t}, 3 \mathrm{H}, J=7.2 \mathrm{~Hz})\end{array}$ & $\begin{array}{l}\text { 164.0, 159.1, 157.8, 152.4, 139.3, 130.0, 126.7, 122.9, 122.2, } \\
114.0,61.6,61.2,55.2,14.1,13.9,12.5\end{array}$ \\
\hline c9 & $\begin{array}{l}7.97(\mathrm{~d}, 1 \mathrm{H}, J=8.0 \mathrm{~Hz}), 7.49(\mathrm{t}, 1 \mathrm{H}, J=7.6 \mathrm{~Hz}), 7.42(\mathrm{t}, 1 \mathrm{H}, J=7.6 \mathrm{~Hz}) \\
7.22(\mathrm{~d}, 1 \mathrm{H}, J=7.6 \mathrm{~Hz}), 4.36(\mathrm{q}, 2 \mathrm{H}, J=6.8 \mathrm{~Hz}), 4.09(\mathrm{q}, 2 \mathrm{H}, J=6.8 \mathrm{~Hz}), \\
3.72(\mathrm{~s}, 3 \mathrm{H}), 2.20(\mathrm{~s}, 3 \mathrm{H}), 1.34(\mathrm{t}, 3 \mathrm{H}, J=7.2 \mathrm{~Hz}), 1.03(\mathrm{t}, 3 \mathrm{H}, J=7.2 \mathrm{~Hz})\end{array}$ & $\begin{array}{l}167.1,162.8,158.0,152.2,140.4,131.9,131.6,131.1,130.5, \\
128.2,126.1,122.7,61.2,61.1,52.1,14.1,13.6,12.2\end{array}$ \\
\hline $\mathrm{c} 10$ & $\begin{array}{l}7.19(\mathrm{~s}, 4 \mathrm{H}), 4.35(\mathrm{q}, 2 \mathrm{H}, J=7.2 \mathrm{~Hz}), 4.25(\mathrm{q}, 2 \mathrm{H}, J=7.2 \mathrm{~Hz}), 2.63(\mathrm{q}, 2 \mathrm{H} \\
J=7.6 \mathrm{~Hz}), 2.38(\mathrm{~s}, 3 \mathrm{H}), 1.33(\mathrm{t}, 3 \mathrm{H}, J=6.8 \mathrm{~Hz}), 1.18-1.25(\mathrm{~m}, 6 \mathrm{H})\end{array}$ & $\begin{array}{l}\text { 164.0, 157.8, 152.5, 143.8, 139.4, 128.7, 128.0, 127.9, 126.7, } \\
122.6,61.6,61.2,28.5,15.3,14.2,13.9,12.6\end{array}$ \\
\hline $\mathrm{c} 11$ & $\begin{array}{l}7.25-7.33(\mathrm{~m}, 2 \mathrm{H}), 7.06-7.15(\mathrm{~m}, 2 \mathrm{H}), 4.35(\mathrm{q}, 2 \mathrm{H}, J=8.0 \mathrm{~Hz}), 4.18(\mathrm{q}, 2 \mathrm{H}, \\
J=8.0 \mathrm{~Hz}), 2.50(\mathrm{q}, 2 \mathrm{H}, J=7.6 \mathrm{~Hz}), 2.30(\mathrm{~s}, 3 \mathrm{H}), 1.33(\mathrm{t}, 3 \mathrm{H}, J=7.2 \mathrm{~Hz}), \\
1.14(\mathrm{t}, 3 \mathrm{H}, J=7.2 \mathrm{~Hz}), 0.99(\mathrm{t}, 3 \mathrm{H}, J=7.2 \mathrm{~Hz})\end{array}$ & $\begin{array}{l}163.1,157.7,153.6,140.5,131.3,129.9,126.3,124.0,118.6, \\
116.8,115.8,61.4,61.3,46.2,14.1,13.7,12.6,11.5\end{array}$ \\
\hline $\mathrm{c} 12$ & $\begin{array}{l}7.30-7.33(\mathrm{~m}, 2 \mathrm{H}), 7.07-7.15(\mathrm{~m}, 2 \mathrm{H}), 4.36(\mathrm{q}, 2 \mathrm{H}, J=7.2 \mathrm{~Hz}), 4.21(\mathrm{q}, 2 \mathrm{H} \\
J=8.0 \mathrm{~Hz}), 2.31(\mathrm{~s}, 3 \mathrm{H}), 1.32(\mathrm{t}, 3 \mathrm{H}, J=7.2 \mathrm{~Hz}), 1.15(\mathrm{t}, 3 \mathrm{H}, J=7.2 \mathrm{~Hz})\end{array}$ & $\begin{array}{l}\text { 163.1, 157.7, 153.6, 140.5, 131.3, 129.9, } 129.8126 .3,124.1, \\
118.6,115.8,115.6,61.4,61.3,14.1,13.8,12.7\end{array}$ \\
\hline $\mathrm{c} 13$ & $\begin{array}{l}\text { 7.33-7.34 (m, 1H), 7.05-7.06 (m, 2H), 4.30-4.39 (m, 4H), } 2.49(\mathrm{~s}, 3 \mathrm{H}) \\
1.28-1.37(\mathrm{~m}, 6 \mathrm{H})\end{array}$ & $\begin{array}{l}\text { 163.7, 157.6, 153.2, 139.5, 132.9, } 127.3126 .9,125.9,116.1, \\
111.6,61.9,61.4,14.2,13.9,13.0\end{array}$ \\
\hline c14 & $\begin{array}{l}7.83(\mathrm{~d}, 2 \mathrm{H}, J=7.2 \mathrm{~Hz}), 7.36-7.44(\mathrm{~m}, 3 \mathrm{H}), 7.26(\mathrm{~d}, 2 \mathrm{H}, J=8.0 \mathrm{~Hz}), 6.93 \\
(\mathrm{~d}, 2 \mathrm{H}, J=7.6 \mathrm{~Hz}), 4.13(\mathrm{q}, 2 \mathrm{H}, J=6.8 \mathrm{~Hz}), 3.85(\mathrm{~s}, 3 \mathrm{H}), 2.32(\mathrm{~s}, 3 \mathrm{H}), 1.05 \\
(\mathrm{t}, 3 \mathrm{H}, J=6.8 \mathrm{~Hz})\end{array}$ & $\begin{array}{l}164.6,158.7,153.7,148.2,130.7,130.2,128.5,128.0,127.5, \\
125.2,122.5,115.0,113.4,60.3,55.2,13.6,11.9\end{array}$ \\
\hline $\mathrm{c} 15$ & $\begin{array}{l}7.45-7.47(\mathrm{~m}, 2 \mathrm{H}), 7.31-7.33(\mathrm{~m}, 3 \mathrm{H}), 3.93(\mathrm{~s}, 3 \mathrm{H}), 3.89(\mathrm{~s}, 3 \mathrm{H}), 2.49(\mathrm{~s}, \\
3 \mathrm{H})\end{array}$ & $\begin{array}{l}162.6,159.4,157.8,140.6,131.6,128.7,128.5,126.4,122.7, \\
106.5,94.9,52.7,52.5,13.2\end{array}$ \\
\hline $\mathrm{c} 16$ & $\begin{array}{l}7.37(\mathrm{~d}, 2 \mathrm{H}, J=8.0 \mathrm{~Hz}), 7.13(\mathrm{~d}, 2 \mathrm{H}, J=7.2 \mathrm{~Hz}), 3.95(\mathrm{~s}, 3 \mathrm{H}), 3.91(\mathrm{~s}, 3 \mathrm{H}) \\
2.50(\mathrm{~s}, 3 \mathrm{H}), 2.36(\mathrm{~s}, 3 \mathrm{H})\end{array}$ & $\begin{array}{l}162.0,158.5,157.2,140.0,138.3,130.9,128.6,125.9,119.1, \\
106.0,94.5,52.0,51.8,20.9,12.5\end{array}$ \\
\hline $\mathrm{c} 17$ & $\begin{array}{l}7.84(\mathrm{~d}, 2 \mathrm{H}, J=8.0 \mathrm{~Hz}), 7.53(\mathrm{~d}, 2 \mathrm{H}, J=8.0 \mathrm{~Hz}), 7.06-7.22(\mathrm{~m}, 9 \mathrm{H}), 2.53 \\
(\mathrm{~s}, 3 \mathrm{H}), 2.38(\mathrm{~s}, 3 \mathrm{H}), 2.31(\mathrm{~s}, 3 \mathrm{H})\end{array}$ & $\begin{array}{l}191.1,154.4,151.8,143.4,138.2,134.8,130.6,129.8,128.6, \\
128.5,127.5,127.4,126.2,126.0,122.7,121.2,105.6,94.5, \\
79.6,21.4,20.7,12.3\end{array}$ \\
\hline c18 & $7.94(\mathrm{~d}, 2 \mathrm{H}, J=7.2 \mathrm{~Hz}), 7.08-7.65(\mathrm{~m}, 13 \mathrm{H}), 2.54(\mathrm{~s}, 3 \mathrm{H})$ & $191.3,154.8,151.9,137.2,132.7,130.7,129.6,128.8,128.4$ \\
\hline
\end{tabular}




\begin{tabular}{|c|c|c|}
\hline Compounds & ${ }^{1} \mathrm{H}$ NMR, $\delta_{H}$ & ${ }^{13} \mathrm{C}$ NMR, $\delta$ \\
\hline & & $\begin{array}{l}128.3,128.2,128.1,127.9,127.8,127.6,127.5,126.7,126.1, \\
122.5,121.7,105.7,94.7,79.4,12.3\end{array}$ \\
\hline $\mathrm{c} 19$ & 7.76-7.78 (m, 2H), 7.20-7.36 (m, 7H), $6.23(\mathrm{~s}, 1 \mathrm{H}), 2.37(\mathrm{~s}, 3 \mathrm{H})$ & $\begin{array}{l}189.8,156.9,151.5,139.7,131.3,130.9,130.0,129.7,129.2, \\
129.1,128.9,128.0,127.9,126.3,122.5,109.0,13.3\end{array}$ \\
\hline $\mathrm{c} 20$ & $\begin{array}{l}7.74(\mathrm{~d}, 2 \mathrm{H}, J=8.0 \mathrm{~Hz}), 7.37-7.43(\mathrm{~m}, 2 \mathrm{H}), 7.22-7.33(\mathrm{~m}, 3 \mathrm{H}), 7.16-7.20 \\
(\mathrm{~m}, 2 \mathrm{H}), 6.46(\mathrm{~s}, 1 \mathrm{H}), 2.43(\mathrm{~s}, 3 \mathrm{H})\end{array}$ & $\begin{array}{l}\text { 191.0, 152.4, 138.0, 133.5, 132.2, 131.9, 130.1, 129.8, 129.7, } \\
129.3,127.8,126.3,124.3,108.2,13.4\end{array}$ \\
\hline $\mathrm{c} 21$ & 7.71-7.73 (m, 2H), 7.42-7.44 (m, 1H), 7.14-7.32 (m, 11H), $2.47(\mathrm{~s}, 3 \mathrm{H})$ & $\begin{array}{l}191.9,150.7,149.1,137.6,133.4,132.5,132.1,132.0,130.1, \\
129.8,129.6,129.4,129.3,128.1,127.7,126.8,126.4,124.2, \\
122.8,12.3\end{array}$ \\
\hline $\mathrm{c} 22$ & 7.70-7.73 (m, 2H), 7.29-7.35 (m, 5H), 7.16-7.25 (m, 7H), $2.37(\mathrm{~s}, 3 \mathrm{H})$ & $\begin{array}{l}191.0,154.5,148.4,138.4,132.6,132.0,131.7,131.0,129.6, \\
129.3,128.9,128.5,128.1,127.9,127.7,127.5,126.8,125.9, \\
123.3,12.0\end{array}$ \\
\hline $\mathrm{c} 23$ & $\begin{array}{l}\text { 7.78-7.80 (m, 2H), 7.63-7.66 (m, 2H), 7.30-7.39 (m, 3H), 7.06-7.08 (m, } \\
1 \mathrm{H}), 6.44(\mathrm{~s}, 1 \mathrm{H}), 2.45(\mathrm{~s}, 3 \mathrm{H})\end{array}$ & $\begin{array}{l}\text { 183.4, 153.6, 151.3, 144.8, 134.2, 133.9, 133.0, 130.8, 130.0, } \\
128.6,128.3,127.8,127.0,121.6,109.3,13.4\end{array}$ \\
\hline $\mathrm{c} 24$ & $\begin{array}{l}\text { 7.88-7.90 (m, 3H), 7.48-7.60 (m, 3H), 7.38-7.40 (m, 1H), 7.08-7.10 (m, } \\
1 \mathrm{H}), 6.29(\mathrm{~s}, 1 \mathrm{H}), 2.41(\mathrm{~s}, 3 \mathrm{H})\end{array}$ & $\begin{array}{l}\text { 190.5, 150.4, 138.9, 132.2, 132.0, 129.3, 128.2, 127.6, 127.3, } \\
127.1,120.3,109.7,13.3\end{array}$ \\
\hline $\mathrm{c} 25$ & $\begin{array}{l}7.87(\mathrm{~d}, 2 \mathrm{H}, J=8.0 \mathrm{~Hz}), 7.82(\mathrm{~d}, 2 \mathrm{H}, J=8.0 \mathrm{~Hz}), 7.74(\mathrm{~d}, 2 \mathrm{H}, J=8.0 \mathrm{~Hz}), \\
7.64(\mathrm{~d}, 2 \mathrm{H}, J=8.0 \mathrm{~Hz}), 7.38-7.43(\mathrm{~m}, 3 \mathrm{H}), 7.30-7.32(\mathrm{~m}, 3 \mathrm{H}), 7.21(\mathrm{~d}, 2 \mathrm{H}, \\
J=8.0 \mathrm{~Hz}), 7.14(\mathrm{~d}, 2 \mathrm{H}, J=8.0 \mathrm{~Hz}), 6.31(\mathrm{~s}, 2 \mathrm{H}), 2.42(\mathrm{~s}, 3 \mathrm{H}), 2.41(\mathrm{~s}, \\
3 \mathrm{H}), 2.39(\mathrm{~s}, 3 \mathrm{H}), 2.34(\mathrm{~s}, 3 \mathrm{H})\end{array}$ & $\begin{array}{l}191.9,191.7,154.9,153.9,151.0,150.8,143.5,138.7,138.4, \\
135.8,132.5,132.3,130.1,129.9,129.6,129.4,129.0,128.9, \\
128.6,128.4,128.3,128.2,128.1,127.3,127.2,127.1,127.1, \\
121.9,121.1,109.8,109.7,21.6,21.3,13.5,13.4\end{array}$ \\
\hline $\mathrm{c} 26$ & $\begin{array}{l}7.85(\mathrm{~d}, 2 \mathrm{H}, J=8.0 \mathrm{~Hz}), 7.80(\mathrm{~d}, 2 \mathrm{H}, J=8.0 \mathrm{~Hz}), 7.62(\mathrm{~d}, 2 \mathrm{H}, J=7.2 \mathrm{~Hz}) \\
7.53(\mathrm{~d}, 2 \mathrm{H}, J=8.0 \mathrm{~Hz}), 7.38-7.43(\mathrm{~m}, 2 \mathrm{H}), 7.25-7.32(\mathrm{~m}, 16 \mathrm{H}), 7.08-9.10 \\
(\mathrm{~m}, 4 \mathrm{H}), 2.50(\mathrm{~s}, 3 \mathrm{H}), 2.49(\mathrm{~s}, 3 \mathrm{H}), 2.32(\mathrm{~s}, 3 \mathrm{H}), 2.31(\mathrm{~s}, 3 \mathrm{H})\end{array}$ & $\begin{array}{l}194.0,193.8,148.3,144.4,138.3,133.3,132.6,132.5,130.2, \\
130.0,129.4,129.3,128.7,128.5,128.4,128.2,127.1,126.4, \\
126.2,123.7,21.8,21.4,12.6,12.5\end{array}$ \\
\hline
\end{tabular}

Table 2. Nano-Cu$u_{2} \mathrm{O}$-catalyzed synthesis of furan derivatives.

\begin{tabular}{|c|c|c|c|c|c|}
\hline Product & $\mathbf{R}^{1}$ & $\mathbf{R}^{2}$ & $\mathbf{R}^{3}$ & $\mathbf{R}^{4}$ & Yield (\%) \\
\hline $\mathrm{c1}$ & $\mathrm{H}$ & $\mathrm{CO}_{2} \mathrm{Et}$ & OEt & $\mathrm{H}$ & 71 \\
\hline $\mathrm{c} 2$ & $\mathrm{CH}_{3}$ & $\mathrm{CO}_{2} \mathrm{Et}$ & OEt & $\mathrm{H}$ & 70 \\
\hline c3 & Et & $\mathrm{CO}_{2} \mathrm{Et}$ & OEt & $\mathrm{H}$ & 64 \\
\hline c4 & $\mathrm{Ph}$ & $\mathrm{CO}_{2} \mathrm{Et}$ & OEt & $\mathrm{H}$ & 68 \\
\hline $\mathrm{c} 5$ & $m-\mathrm{CH}_{3} \mathrm{Ph}$ & $\mathrm{CO}_{2} \mathrm{Et}$ & OEt & $\mathrm{H}$ & 68 \\
\hline c6 & $o-\mathrm{CH}_{3} \mathrm{Ph}$ & $\mathrm{CO}_{2} \mathrm{Et}$ & OEt & $\mathrm{H}$ & 59 \\
\hline c7 & $p-\mathrm{CH}_{3} \mathrm{CO}_{2} \mathrm{Ph}$ & $\mathrm{CO}_{2} \mathrm{Et}$ & OEt & $\mathrm{H}$ & 69 \\
\hline $\mathrm{c} 8$ & $p-\mathrm{CH}_{3} \mathrm{OPh}$ & $\mathrm{CO}_{2} \mathrm{Et}$ & OEt & $\mathrm{H}$ & 66 \\
\hline c9 & $o-\mathrm{CH}_{3} \mathrm{OPh}$ & $\mathrm{CO}_{2} \mathrm{Et}$ & OEt & $\mathrm{H}$ & 55 \\
\hline $\mathrm{c} 10$ & $p$-EtPh & $\mathrm{CO}_{2} \mathrm{Et}$ & OEt & $\mathrm{H}$ & 67 \\
\hline c11 & $o$-EtPh & $\mathrm{CO}_{2} \mathrm{Et}$ & OEt & $\mathrm{H}$ & 53 \\
\hline $\mathrm{c} 12$ & $o-\mathrm{FPh}$ & $\mathrm{CO}_{2} \mathrm{Et}$ & OEt & $\mathrm{H}$ & 62 \\
\hline c13 & 2-thienyl & $\mathrm{CO}_{2} \mathrm{Et}$ & OEt & $\mathrm{H}$ & 64 \\
\hline c14 & $p-\mathrm{CH}_{3} \mathrm{OPh}$ & $\mathrm{Ph}$ & OEt & $\mathrm{H}$ & 79 \\
\hline c15 & $p$-phenylethynyl & $\mathrm{CO}_{2} \mathrm{Me}$ & $\mathrm{OMe}$ & $\mathrm{H}$ & 70 \\
\hline c16 & $p$-tolylethynyl & $\mathrm{CO}_{2} \mathrm{Me}$ & $\mathrm{OMe}$ & $\mathrm{H}$ & 68 \\
\hline c17 & p-phenylethynyl & $p-\mathrm{CH}_{3} \mathrm{Ph}$ & $p-\mathrm{CH}_{3} \mathrm{Ph}$ & $\mathrm{H}$ & 75 \\
\hline $\mathrm{c} 18$ & $p$-phenylethynyl & $\mathrm{Ph}$ & $\mathrm{Ph}$ & $\mathrm{H}$ & 72 \\
\hline c19 & $\mathrm{H}$ & $\mathrm{Ph}$ & $o$-chlorophenyl & $\mathrm{H}$ & 37 \\
\hline $\mathrm{c} 20$ & $\mathrm{H}$ & $o$-chlorophenyl & $\mathrm{Ph}$ & $\mathrm{H}$ & 37 \\
\hline $\mathrm{c} 21$ & $\mathrm{Ph}$ & $\mathrm{Ph}$ & $o$-chlorophenyl & $\mathrm{H}$ & 29 \\
\hline $\mathrm{c} 22$ & $\mathrm{Ph}$ & $o$-chlorophenyl & $\mathrm{Ph}$ & $\mathrm{H}$ & 35 \\
\hline $\mathrm{c} 23$ & $\mathrm{H}$ & $\mathrm{Ph}$ & 2-thienyl & $\mathrm{H}$ & 36 \\
\hline c24 & $\mathrm{H}$ & 2-thienyl & $\mathrm{Ph}$ & $\mathrm{H}$ & 36 \\
\hline $\mathrm{c} 25$ & $p-\mathrm{CH}_{3} \mathrm{Ph}$ & $\mathrm{Ph}$ & $\mathrm{H}$ & $\mathrm{H}$ & 44 \\
\hline c26 & $\mathrm{Ph}$ & $p-\mathrm{CH}_{3} \mathrm{Ph}$ & $\mathrm{Ph}$ & $\mathrm{H}$ & 20 \\
\hline
\end{tabular}

\subsection{Fungicidal Activity}

All the compounds were evaluated for the fungicidal activities against five phytopathogenic fungi and the results were listed in Table 3. In general, the fungicidal activities screening results indicated that the fungicidal activities of $\mathrm{c} 7$, c8, c10 against $F$. oxysporum and $A$. solani, and that of c15, c17, c18 against $F$. graminearum, $R$. solani and $V$. mali were considerable at the concentration of $50 \mathrm{mg} / \mathrm{L}$ with growth inhibition rate of more than $50 \%$.

Among them, except $F$. oxysporum, all compounds showed weaker fungicidal activities against the other four tested fungi than that of the commercial fungicide chlorothalonil. However, c14 and c15 exhibited much higher fungicidal activities against F. oxysporum than that of chlorothalonil with inhibitory rate of $73.64 \%$ and $79.86 \%$, respectively, especially, c14 only obviously inhibited the mycelia growth of $F$. oxysporum, which showed its fungicidal spectrum might be too specific. 
Table 3. Fungicidal activities of the tested compounds in vitro $(50 \mathrm{mg} / \mathrm{L})$.

\begin{tabular}{lllll}
\hline Compounds & F.oxysporum & A. solani & F.graminearum & R. solani \\
\hline c7 & $68.92 \pm 0.10$ & $51.54 \pm 0.09$ & $37.43 \pm 0.19$ & $33.37 \pm 0.35$ \\
c8 & $62.43 \pm 0.06$ & $59.89 \pm 0.07$ & $32.04 \pm 0.04$ & $42.56 \pm 0.17$ \\
c10 & $65.85 \pm 0.25$ & $62.51 \pm 0.17$ & $40.67 \pm 0.15$ & $39.20 \pm 0.16$ \\
c14 & $73.64 \pm 0.10$ & $40.55 \pm 0.11$ & $37.42 \pm 0.18$ & $41.17 \pm 0.70$ \\
c15 & $79.86 \pm 0.25$ & $64.43 \pm 0.25$ & $55.31 \pm 0.11$ & $61.94 \pm 0.04$ \\
c17 & $68.36 \pm 0.18$ & $52.51 \pm 0.20$ & $61.44 \pm 0.14$ & $55.73 \pm 0.16$ \\
c18 & $69.39 \pm 0.28$ & $54.28 \pm 0.19$ & $58.65 \pm 0.26$ & $55.08 \pm 0.11$ \\
Chlorothalonil & $66.85 \pm 0.31$ & $72.23 \pm 0.35$ & $76.66 \pm 0.19$ & 87.31 \\
\hline
\end{tabular}

Thus, $F$. oxysporum was chosen as the further tested fungi to determine the toxicity regression equation and median effective concentration $\left(\mathrm{EC}_{50}\right)$ of the target compounds in order to provide a reference for structure-activities relationship study, and the results were showed in Table 4. The bioassay results indicated that $\mathrm{c} 14$ and $\mathrm{c} 15$ had appreciable inhibitory effect on $F$. oxysporum with $\mathrm{EC}_{50}$ value of 14.71 $\mathrm{mg} / \mathrm{L}$ and $14.39 \mathrm{mg} / \mathrm{L}$, respectively, which was superior to that of positive control with $\mathrm{EC}_{50}$ value of $28.15 \mathrm{mg} / \mathrm{L}$. Meanwhile, $\mathrm{EC}_{50}$ values of the other compounds except c14 and c15 ranged from $20.74 \mathrm{mg} / \mathrm{L}$ to $31.43 \mathrm{mg} / \mathrm{L}$, which was equal or similar to that of chlorothalonil. In summary, compared with the other compounds, c15 exhibited the strongest fungicidal activities against four phytopathogenic fungi except $F$. graminearum. Recently, the researches of novel furans derivatives mainly focus on its potential biological activities against clinical bacteria and food microorganism, such as $\alpha$-fury lacrylic acid [22] and 5-hydroxymethyl furan-3-carboxylic acid [23], but rarely on fungicidal activities, which indicated that it is quite important to further explore its fungicidal mechanism. However, all synthetic furans compounds in this study used as candidate clinical antibacterial medicine or food preservatives also require further study and measurement.

Table 4. The $E C_{50}$ value of tested compounds against $F$. oxysporum.

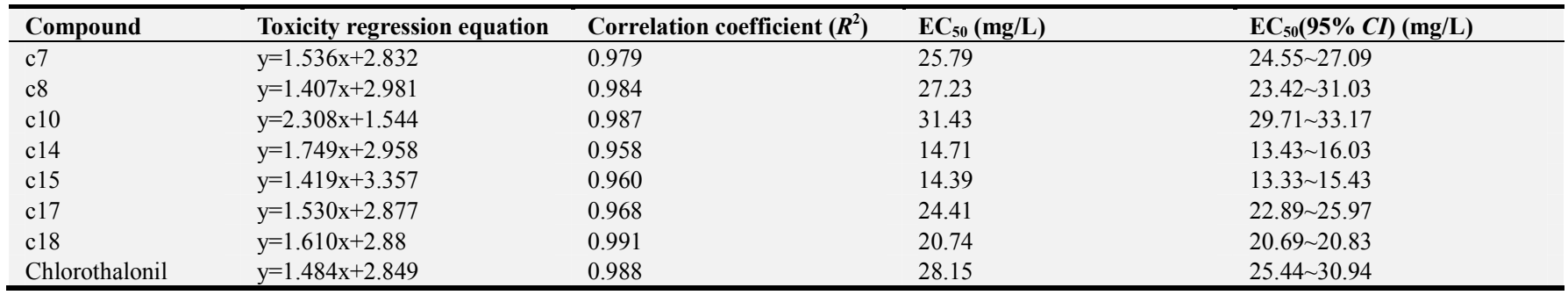

\section{Conclusions}

A series of furan derivatives were synthesized and the research provided a convenient strategy for synthesis of novel substituted-furans derivatives. The bioassay results indicated that $\mathrm{c} 14$ and $\mathrm{c} 15$ exhibited higher fungicidal activities against $F$. oxysporum than chlorothalonil. Among the synthesized compounds, c15, c17 and c18 could inhibit the mycelium growth of five phytopathogenic fungi effectively, had more wider fungicidal spectrum than the other compounds. Especially, c15 exhibited more stronger fungicidal activities against $F$. oxysporum, $A$. solani, $R$. solani and $V$. mali than the other compounds. In conclusion, $\mathrm{c} 15$ has great research value and broad application prospect as potential lead structure for structural optimization.

\section{Acknowledgements}

This work was supported by the Training Plan for Outstanding Young Teachers in Higher Education Institutions of Guangdong Province (No. YQ2015203), the National Natural Science Foundation of China (No. 21302023), Talent
Fund Project of Guangdong Vocational College of Environmental Protection Engineering (K660116011812).

\section{References}

[1] R. C. D. Brown, Developments in furan syntheses, Angew. Chem. Int. Ed. 2005, 44, pp. 850-852.

[2] S. F. Kirsch, Syntheses of polysubstituted furans: recent developments, Org. Biomol. Chem. 2006, 4, pp. 2076-2080.

[3] D. Kalaitzakis, M. Triantafyllakis, I. Alexopoulou, et al., One-pot transformation of simple furans into 4-hydroxy-2-cyclopentenones in water, Angew. Chem. Int. Ed. 2014, 53, pp. 13201-13205.

[4] Y. J. Shi, S. L. Wang, H. B. He, et al., Synthesis and bioactivity of novel pyrazole oxime ester derivatives containing furan moiety, Chin. J. Org. Chem. 2015, 35, pp. 1785-1791.

[5] F. Hasegawa, K. Niidome, C. Migihashi, et al., Discovery of furan-2-carbohydrazides as orally active glucagon receptor antagonists, Bioorg. Med. Chem. Lett. 2014, 24, pp. 4266-4270.

[6] J. Q. Huo, L. Y. Ma, Z. Zhang, et al., Synthesis and biological activity of novel N-(3-furan-2-yl-1-phenyl-1H-pyrazol-5-yl) amides derivatives, Chin. Chem. Lett. 2016, 9, pp. 1547-1550. 
[7] Kobeissy A A, Hashash J G, Jamali F R, Skoury A M, Haddad R, El-Samad S, Ladki R, Aswad R, Soweid A M, World J Gastroenterol, 2012, 19, 2390-2395.

[8] J. P. Gisbert, L. Gonzalez, X. Calvet, Systematic review and meta-analysis: proton pump inhibitor vs. ranitidine bismuth citrate plus two antibiotics in Helicobacter pylori eradication, Helicobacter, 2005, 10, pp. 157-171.

[9] B. Mahesh, B. Yim, D. Robson, et al., Does furosemide prevents renal dysfunction in high-risk cardiac surgical patients? Result of a double-blinded prospective randomised trial, Eur. J. Cardiothorac. Surg. 2008, 33, pp. 370-376.

[10] B. Meryem, A. W. Noori, E. H. Redouan, et al., Comparison of hypotensive, diuretic and renal effects between cladodes of Opuntia ficus-indica and furosemide, Asian Pac. J. Trop. Med. 2017, 9, pp. 900-906.

[11] Y. Xie, Y. Zhu, H. Zhou, et al., Furazolidone-based triple and quadruple eradication therapy for Helicobacter pylori infection, World J. Gastroenterol. 2014, 32, pp. 11415-11421.

[12] M. Zamani, A. Rahbar, J. Shokri-Shirvani. Resistance of Helicobacter pylori to furazolidone and levofloxacin: A viewpoint, World J. Gastroenterol. 2017, 37, pp. 6920-6922.

[13] M. Vass, K. Hruska, M. Franek, Nitrofuran antibiotics: a review on the application, prohibition and residual analysis, Vet. Med. Czech, 2008, 9, pp. 469-500.

[14] T. Alarcón, P. de la Obra, D. Domingo, et al., In vitro activity of furazolidone and nitrofurantoin in Helicobacter pylori clinical isolates and study of mutation rate. Rev. Esp. Quim. 2005, 18, pp. 313-318.

[15] Rosenthal A, Neter E, Weppner D F, Recovery from Salmonella meningitis after treatment with furaltadone (Altafur), J Pediatr, 1961, 3, pp. 377.
[16] G. J. Liu, R. M. Wilkins, Lethal and anitfeeding effects of carbofuran against the whitebacked planthopper, Chin. J. Rcie Sci. 1992, 3, pp. 119-124.

[17] L. L. Peng, X. Zhang, J. Ma, Synthesis of furan from allenic sulfide derivatives, Sci. China Chem. 2009, 10, pp. 1622-1630.

[18] J. M. Liu, X. Y. Liu, X. S. Qing, et al., $\mathrm{I}_{2} / \mathrm{K}_{2} \mathrm{CO}_{3}$-promoted ring-opening/cyclization/ rearrangement/aromatization sequence: A powerful strategy for the synthesis of polysubstituted furans, Chin. Chem. Lett. 2017, 2, pp. 458462.

[19] M. Miao, X. Xu, L. Xu, Copper (I) iodide mediated iodocyclization of cyclopropylideneallenyl ketones: facile and effective synthesis of highly substituted furan derivatives, Eur. J. Org. Chem. 2014, 27, pp. 5896-5900.

[20] C. He, S. Guo, J. Ke, et al., Silver-mediated oxidative C-H/C$\mathrm{H}$ functionalization: a strategy to construct polysubstituted furans, J. Am. Chem. Soc. 2012, 134, pp. 5766-5769.

[21] J. Wu, N. Yoshikai, Modular synthesis of multisubstituted furans through palladium-catalyzed three-component condensation of alkynylbenziodoxoles carboxylic acids, and imines, Angew. Chem. Int. Ed. 2015, 54, pp. 11107-11111.

[22] H. Yu, Z. Ning, Y. Li, J. Gao, Preparation and antimicrobial activity of $\alpha$-fury lacrylic acid, J. Anhui Agr. Univ., 2006, 3, pp. 351-355.

[23] C. Ma, Y. Ma, T. Li, J. Wang, Structure and activity of secondary metabolites of Endophytic fungus S19 strain in Cephalotaxus fortunei, Guizhou Agr. Sci., 2014, 12, pp. 152-156. 